\title{
On Interval-Valued Pseudolinear Functions and Interval-Valued Pseudolinear Optimization Problems
}

\author{
Jianke Zhang, ${ }^{1,2}$ Qinghua Zheng, ${ }^{1}$ Chang Zhou, ${ }^{2}$ Xiaojue $\mathrm{Ma}^{2}$ and Lifeng $\mathrm{Li}^{2}$ \\ ${ }^{1}$ Department of Computers, Xian Jiaotong University, Xian 710049, China \\ ${ }^{2}$ Department of Mathematics, School of Science, Xian University of Posts and Telecommunications, Xian 710121, China \\ Correspondence should be addressed to Jianke Zhang; jiankezh@163.com
}

Received 23 January 2015; Revised 12 March 2015; Accepted 17 March 2015

Academic Editor: Adrian Petrusel

Copyright (c) 2015 Jianke Zhang et al. This is an open access article distributed under the Creative Commons Attribution License, which permits unrestricted use, distribution, and reproduction in any medium, provided the original work is properly cited.

\begin{abstract}
Some basic characterizations of an interval-valued pseudolinear function are derived. By means of the properties of intervalvalued pseudolinearity, a class of interval-valued pseudolinear programs is considered, and the solution set of the interval-valued pseudolinear optimization problem is characterized.
\end{abstract}

\section{Introduction}

In recent years, many approaches for interval-valued optimization problems have been explored in considerable details; see examples in [1-3]. Several authors have been interested in the optimality conditions and duality results for the interval-valued optimization problems. Wu has extended the concept of convexity for real-valued functions to LUconvexity for interval-valued functions, and then he has established the Karush-Kuhn-Tucker (KKT) optimality conditions [4] and duality theory [5] for an optimization problem with an interval-valued objective function under the assumption of LU-convexity. Sun and Wang [6] have derived the Fritz John type and Kuhn-Tucker type necessary and sufficient optimality conditions for nondifferentiable interval-valued programming. Jayswal et al. [7] have discussed Mond-Weir and Wolfe type duality theorems for the interval-valued programming problems under the conditions of generalized convexity. Chalco-Cano et al. [8] have obtained KKT optimality conditions for interval-valued programming problems by using a more general concept of gH-derivative. Bhurjee and Panda $[9,10]$ have introduced the parametric form of interval-valued functions and studied the solution of convex interval-valued programming problems. Jana and Panda [11] have studied the preferable efficient solutions of the problem of interval-valued vector optimization. Zhang et al. [12] have extended the concepts of preinvexity and invexity to intervalvalued functions and derived the KKT optimality conditions for LU-preinvex and invex optimization problems with an interval-valued objective function.

Interval-valued linear optimization $[13,14]$ is a class of important and simple interval-valued optimization problems. Recently, Hladík [15] has proposed a method for testing basis stability of interval-valued linear optimization problems; Hladík's studies have shown that if some basis stability criterion holds true, then the problems become much more easy to solve. In [16], Hladík has discussed lower and upper bound approximation for the best case optimal value of the interval-valued linear optimization problems and proposed an algorithm for computing the best case optimal value. Li et al. [17, 18] and Luo et al. [19] have established some necessary and sufficient conditions for checking weak and strong optimality of given feasible solutions for the intervalvalued linear optimization problems.

In this paper, we consider and give some characterizations for a class of generalized interval-valued linear function, which is interval-valued pseudolinear function. Then, by means of the basic properties of interval-valued pseudolinearity, a class of interval-valued pseudolinear programs is considered, and the solution set of the interval-valued pseudolinear optimization problem is characterized. It can be shown that the interval-valued linear optimization problems 
$[13,14]$ and the interval-valued linear fractional programs are included in interval-valued pseudolinear programs. These programs arise in many practical applications.

\section{Preliminaries}

In this section, we recall some basic concepts with regard to interval-valued functions.

Let $R^{n}$ be $n$-dimensional Euclidean space, and let $R_{+}^{n}$ be its nonnegative orthant. Let us denote by $\mathscr{I}$ the class of all closed intervals in $R . A=\left[a^{L}, a^{U}\right] \in \mathscr{I}$ denotes a closed interval, where $a^{L}$ and $a^{U}$ mean the lower and upper bounds of $A$, respectively. The closed interval $A$ also can be expressed in terms of a parameter in several ways. Throughout this paper, we consider specific parametric representations of the interval as $A=\left[a^{L}, a^{U}\right]=\left\{a(t)=a^{L}+t\left(a^{U}-a^{L}\right) \mid t \in[0,1]\right\}$.

Let $A=\left[a^{L}, a^{U}\right]=\left\{a\left(t_{1}\right) \mid t_{1} \in[0,1]\right\}$ and $B=\left[b^{L}, b^{U}\right]=$ $\left\{b\left(t_{2}\right) \mid t_{2} \in[0,1]\right\}$ be in $\mathscr{I}$, and the interval operations can be performed with respect to parameters $[9,10]$ as follows:

(a) $A \oplus B=\left\{a\left(t_{1}\right)+b\left(t_{2}\right) \mid t_{1}, t_{2} \in[0,1]\right\}$;

(b) $A \ominus B=\left\{a\left(t_{1}\right)-b\left(t_{2}\right) \mid t_{1}, t_{2} \in[0,1]\right\}$;

(c) $A \odot B=\left\{a\left(t_{1}\right) \cdot b\left(t_{2}\right) \mid t_{1}, t_{2} \in[0,1]\right.$, and $k A=\left\{k a\left(t_{1}\right) \mid\right.$ $\left.t_{1} \in[0,1]\right\}$

(d) $A \oslash B=\left\{a\left(t_{1}\right) / b\left(t_{2}\right) \mid t_{1}, t_{2} \in[0,1], b\left(t_{2}\right) \neq 0\right\}$.

Let $\mathbf{C}_{v}^{k}$ be $k$ dimensional column whose elements are intervals. That is, $\mathbf{C}_{v}^{k} \in \mathscr{G}^{k}, \mathbf{C}_{v}^{k}=\left(\mathbf{C}_{1}, \mathbf{C}_{2}, \ldots, \mathbf{C}_{k}\right)^{T}$, and $\mathbf{C}_{j}=$ $\left[c_{j}^{L}, c_{j}^{U}\right]=c_{j}^{L}+t_{j}\left(c_{j}^{U}-c_{j}^{L}\right), t_{j} \in[0,1], j=1,2, \ldots, k$. The interval-valued function in parametric form is as follows.

Definition 1 (see [9]). For $c(t)=\left(c_{1}\left(t_{1}\right), c_{2}\left(t_{2}\right), \ldots, c_{k}\left(t_{k}\right)\right)^{T} \in$ $\mathbf{C}_{v}^{k}$, let $f_{c(t)}: R^{n} \rightarrow R$. For a given interval vector $\mathbf{C}_{v}^{k}$, an interval-valued function $\mathbf{F}_{\mathbf{C}_{v}^{k}}: R^{n} \rightarrow \mathscr{I}$ is defined as

$$
\mathbf{F}_{\mathbf{C}_{v}^{k}}(\mathbf{x})=\left\{f_{c(t)}(\mathbf{x}) \mid f_{c(t)}: R^{n} \longrightarrow R, c(t) \in \mathbf{C}_{v}^{k}\right\} .
$$

For every fixed $\mathbf{x}$, if $f_{c(t)}(\mathbf{x})$ is continuous in $t$, then $\min _{t \in[0,1]^{k}} f_{c(t)}(\mathbf{x})$ and $\max _{t \in[0,1]^{k}} f_{c(t)}(\mathbf{x})$ exist. In that case

$$
\mathbf{F}_{\mathbf{C}_{v}^{k}}(\mathbf{x})=\left[\min _{t \in[0,1]^{k}} f_{c(t)}(\mathbf{x}), \max _{t \in[0,1]^{k}} f_{c(t)}(\mathbf{x})\right] .
$$

If $f_{c(t)}(\mathbf{x})$ is linear in $t$, then $\min _{t \in[0,1]^{k}} f_{c(t)}(\mathbf{x})$ and $\max _{t \in[0,1]^{k}} f_{c(t)}(\mathbf{x})$ exist in the set of vertices of $\mathbf{C}_{v}^{k}$. If $f_{c(t)}(\mathbf{x})$ is monotonically increasing in $t$, then $\mathbf{F}_{\mathbf{C}_{v}^{k}}(\mathbf{x})=$ $\left[f_{c(0)}(\mathbf{x}), f_{c(1)}(\mathbf{x})\right]$.

The interval-valued function $\mathbf{F}_{\mathrm{C}_{v}^{k}}: R^{n} \rightarrow \mathscr{I}$ is differentiable at $\mathbf{x}^{*}$ if $f_{c(t)}(\mathbf{x})$ is differentiable at $\mathbf{x}^{*}$ for every $t \in$ $[0,1]^{k}$. The partial derivatives of $\mathbf{F}_{\mathbf{C}_{v}^{k}}$ at $\mathbf{x}^{*}$ may be calculated as follows:

$$
\begin{aligned}
& \frac{\partial \mathbf{F}_{\mathbf{C}_{v}^{k}}\left(\mathbf{x}^{*}\right)}{\partial x_{i}} \\
& \quad=\left\{\frac{\partial f_{c(t)}\left(\mathbf{x}^{*}\right)}{\partial x_{i}} \mid \text { for every } t \in[0,1]^{k}, c(t) \in \mathbf{C}_{v}^{k}\right\} .
\end{aligned}
$$

If $f_{c(t)}\left(\mathbf{x}^{*}\right)$ is continuous in $t$, then

$$
\begin{aligned}
& \frac{\partial \mathbf{F}_{\mathbf{C}_{v}^{k}}\left(\mathbf{x}^{*}\right)}{\partial x_{i}} \\
& \quad=\left[\min _{t \in[0,1]^{k}} \frac{\partial f_{c(t)}\left(\mathbf{x}^{*}\right)}{\partial x_{i}}, \max _{t \in[0,1]^{k}} \frac{\partial f_{c(t)}\left(\mathbf{x}^{*}\right)}{\partial x_{i}}\right] .
\end{aligned}
$$

The gradient of $\mathbf{F}_{\mathbf{C}_{v}^{k}}$ at $\mathbf{x}^{*}$ is an interval vector:

$$
\begin{aligned}
& \nabla \mathbf{F}_{\mathbf{C}_{v}^{k}}\left(\mathbf{x}^{*}\right) \\
& \quad=\left(\frac{\partial \mathbf{F}_{\mathbf{C}_{v}^{k}}\left(\mathbf{x}^{*}\right)}{\partial x_{1}}, \frac{\partial \mathbf{F}_{\mathbf{C}_{v}^{k}}\left(\mathbf{x}^{*}\right)}{\partial x_{2}}, \ldots, \frac{\partial \mathbf{F}_{\mathbf{C}_{v}^{k}}\left(\mathbf{x}^{*}\right)}{\partial x_{n}}\right)^{T} .
\end{aligned}
$$

In this paper, we denote the point $\lambda \mathbf{y}+(1-\lambda) \mathbf{x}, \lambda \in[0,1]$, by $\mathbf{x} \lambda \mathbf{y}$. For the two interval-valued functions of $\mathbf{F}_{\mathrm{C}_{v}^{k}}(\mathbf{x})=$ $\left\{f_{c\left(t_{1}\right)}(\mathbf{x}) \mid f_{c\left(t_{1}\right)}: R^{n} \rightarrow R, c\left(t_{1}\right) \in \mathbf{C}_{v}^{k}\right\}$ and $\mathbf{G}_{\mathbf{C}_{v}^{k}}(\mathbf{x})=$ $\left\{g_{c\left(t_{2}\right)}(\mathbf{x}) \mid g_{c\left(t_{2}\right)}: R^{n} \rightarrow R, c\left(t_{2}\right) \in \mathbf{C}_{v}^{k}\right\}$, we say that $\mathbf{F}_{\mathbf{C}_{v}^{k}}(\mathbf{x})=$ $\mathbf{G}_{\mathbf{C}_{v}^{k}}(\mathbf{x})$ or $\mathbf{F}_{\mathbf{C}_{v}^{k}}(\mathbf{x}) \leq \mathbf{G}_{\mathbf{C}_{v}^{k}}(\mathbf{x})$, which means

$$
\begin{array}{r}
f_{c\left(t_{1}\right)}(\mathbf{x})=g_{c\left(t_{2}\right)}(\mathbf{x}) \\
\text { or } f_{c\left(t_{1}\right)}(\mathbf{x}) \leq g_{c\left(t_{2}\right)}(\mathbf{x})
\end{array}
$$

for all $t_{1}, t_{2} \in[0,1]^{k}$ and $t_{1}$ may or may not be equal to $t_{2}$.

However, we say that $\mathbf{F}_{\mathbf{C}_{v}^{k}}(\mathbf{x})={ }_{w} \mathbf{G}_{\mathbf{C}_{v}^{k}}(\mathbf{x})$ or $\mathbf{F}_{\mathbf{C}_{v}^{k}}(\mathbf{x}) \unlhd_{w}$ $\mathbf{G}_{\mathrm{C}_{v}^{k}}(\mathbf{x})$, which means

$$
\begin{aligned}
f_{c\left(t_{1}\right)}(\mathbf{x}) & =g_{c\left(t_{2}\right)}(\mathbf{x}) \\
\text { or } f_{c\left(t_{1}\right)}(\mathbf{x}) & \leq g_{c\left(t_{2}\right)}(\mathbf{x})
\end{aligned}
$$

for all $t_{1}=t_{2} \in[0,1]^{k}$; that is to say, $t_{1}$ and $t_{2}$ are the same in both sides.

Definition 2 (see [9]). Suppose that $X \subseteq R^{n}$ is a convex set and for given $\mathbf{C}_{v}^{k} \in \mathscr{I}^{k}, \mathbf{F}_{\mathbf{C}_{v}^{k}}: R^{n} \rightarrow \mathscr{I}$. For $\mathbf{x}_{1}, \mathbf{x}_{2} \in X$, $0 \leq \lambda \leq 1$.

(i) $\mathbf{F}_{\mathbf{C}_{v}^{k}}$ is said to be convex with respect to $\preceq$ if

$$
\mathbf{F}_{\mathbf{C}_{v}^{k}}\left(\lambda \mathbf{x}_{1}+(1-\lambda) \mathbf{x}_{2}\right) \leq \lambda \mathbf{F}_{\mathbf{C}_{v}^{k}}\left(\mathbf{x}_{1}\right) \oplus(1-\lambda) \mathbf{F}_{\mathbf{C}_{v}^{k}}\left(\mathbf{x}_{2}\right),
$$

which means

$$
\begin{aligned}
& f_{c\left(t^{\prime}\right)}\left(\lambda \mathbf{x}_{1}+(1-\lambda) \mathbf{x}_{2}\right) \\
& \quad \leq \lambda f_{c\left(t^{\prime \prime}\right)}\left(\mathbf{x}_{1}\right)+(1-\lambda) f_{c\left(t^{\prime \prime}\right)}\left(\mathbf{x}_{2}\right)
\end{aligned}
$$

for all $t^{\prime}, t^{\prime \prime} \in[0,1]^{k} ; t^{\prime}$ may or may not be equal to $t^{\prime \prime}$.

(ii) $\mathbf{F}_{\mathbf{C}_{v}^{k}}$ is said to be convex with respect to $\unlhd_{w}$ if

$$
\begin{aligned}
& \mathbf{F}_{\mathbf{C}_{v}^{k}}\left(\lambda \mathbf{x}_{1}+(1-\lambda) \mathbf{x}_{2}\right) \\
& \quad{ }_{w} \lambda \mathbf{F}_{\mathbf{C}_{v}^{k}}\left(\mathbf{x}_{1}\right) \oplus(1-\lambda) \mathbf{F}_{\mathbf{C}_{v}^{k}}\left(\mathbf{x}_{2}\right),
\end{aligned}
$$


which means

$$
f_{c(t)}\left(\lambda \mathbf{x}_{1}+(1-\lambda) \mathbf{x}_{2}\right) \leq \lambda f_{c(t)}\left(\mathbf{x}_{1}\right)+(1-\lambda) f_{c(t)}\left(\mathbf{x}_{2}\right)
$$

for all $t \in[0,1]^{k} ; t$ is same in both sides.

It can be shown that $\mathbf{F}_{\mathrm{C}_{v}^{k}}$ is convex [9] with respect to $\preceq_{w}$ if and only if $f_{c(t)}(\mathbf{x})$ is a convex function on $X$ for every $t$. Similar result does not hold for convexity with respect to $\preceq$.

Mangasarian [20] has introduced the following pseudoconvex real-valued functions.

Definition 3 (see [20]). The differentiable real-valued function $f: X \rightarrow R$ on the open set $X \subseteq R^{n}$ is pseudoconvex on $X$ if

$$
\mathbf{x}, \mathbf{y} \in X, \quad(\mathbf{x}-\mathbf{y})^{T} \nabla f(\mathbf{y}) \geq 0 \Longrightarrow f(\mathbf{x})-f(\mathbf{y}) \geq 0 ;
$$

or equivalently,

$$
\mathbf{x}, \mathbf{y} \in X, \quad f(\mathbf{x})-f(\mathbf{y})<0 \Longrightarrow(\mathbf{x}-\mathbf{y})^{T} \nabla f(\mathbf{y})<0 .
$$

Furthermore, we say that $f$ is strictly pseudoconvex on $X$ if

$$
\mathbf{x}, \mathbf{y} \in X, \quad f(\mathbf{x})-f(\mathbf{y}) \leq 0 \Longrightarrow(\mathbf{x}-\mathbf{y})^{T} \nabla f(\mathbf{y})<0 .
$$

We can also define the following pseudoconvex intervalvalued function.

Definition 4. A differentiable interval-valued function $\mathbf{F}_{\mathrm{C}_{v}^{k}}$ : $X \rightarrow \mathscr{I}$, on the convex set $X \subseteq R^{n}$ for given $\mathbf{C}_{v}^{k} \in \mathscr{I}^{k}$, is pseudoconvex with respect to $\preceq_{w}$ on $X$ if

$\mathbf{x}, \mathbf{y} \in X$,

$$
(\mathbf{x}-\mathbf{y})^{T} \nabla \mathbf{F}_{\mathbf{C}_{v}^{k}}(\mathbf{y}) \succeq_{w} 0 \Longrightarrow \mathbf{F}_{\mathbf{C}_{v}^{k}}(\mathbf{x}) \succeq_{w} \mathbf{F}_{\mathbf{C}_{v}^{k}}(\mathbf{y}) ;
$$

or equivalently,

$$
\mathbf{F}_{\mathbf{C}_{v}^{k}}(\mathbf{x}) \prec{ }_{w} \mathbf{F}_{\mathbf{C}_{v}^{k}}(\mathbf{y}) \Longrightarrow(\mathbf{x}-\mathbf{y})^{T} \nabla \mathbf{F}_{\mathbf{C}_{v}^{k}}(\mathbf{y}) \prec{ }_{w} 0 .
$$

An interval-valued function $\mathbf{F}_{\mathbf{C}_{v}^{k}}$ is pseudoconcave if $-\mathbf{F}_{\mathbf{C}_{v}^{k}}$ is pseudoconvex with respect to $\preceq_{w}$ on $X$.

Remark 5. It is worth pointing out that the definition of pseudoconvex interval-valued function in [4] has some limitations, which define the pseudoconvex interval-valued function by using the endpoint functions $f^{L}$ and $f^{U}$ of the interval-valued function $f(\mathbf{x})=\left[f^{L}, f^{U}\right]$. However, in some case, if the two endpoint functions $f^{L}$ and $f^{U}$ are all realvalued pseudoconvex functions, the interval-valued function may be not a pseudoconvex interval-valued function, which can be seen in the following example.

Example 6. The interval-valued function $f(x)=\left[f^{L}(x)\right.$, $\left.f^{U}(x)\right], x \in(0,1), f^{L}(x)=\min \left\{f_{1}(x), f_{2}(x)\right\}$, and $f^{U}(x)=$ $\max \left\{f_{1}(x), f_{2}(x)\right\}$, where $f_{1}(x)=\log x$ and $f_{2}(x)=-2 x^{2}$.

It can be shown that $f_{1}(x)=\log x$ is strictly increasing function, being $\left(f_{1}(x)\right)^{\prime}=1 / x>0$, for all $x \in(0,1)$; therefore, $(y-x)\left(f_{1}(x)\right)^{\prime} \geq 0 \Leftrightarrow y-x \geq 0$. Thus, $y-x \geq$ $0 \Leftrightarrow y \geq x \Rightarrow f_{1}(y) \geq f_{1}(x)$. So $f_{1}(x)$ is pseudoconvex.

The function $f_{2}(x)=-2 x^{2}$ is strictly decreasing on $(0,1)$, as $\left(f_{2}(x)\right)^{\prime}=-4 x<0$. We have $(y-x)\left(f_{2}(x)\right)^{\prime} \geq 0 \Leftrightarrow$ $y-x \leq 0 ; y-x \leq 0 \Leftrightarrow y \leq x \Rightarrow f_{2}(x) \leq f_{2}(y)$, so $f_{2}(x)$ is pseudoconvex.

The sum is $z(x)=\lambda f^{L}(\mathbf{x})+\left.(1-\lambda) f^{U}(\mathbf{x})\right|_{\lambda=1 / 2} \in$ $\left[f^{L}(x), f^{U}(x)\right]$ and $z(x)=(1 / 2)\left(\log x-2 x^{2}\right), 1>x>0$. We have $z^{\prime}(x)=(1 / 2)\left(\log x-2 x^{2}\right)^{\prime}=\left(1-4 x^{2}\right) / 2 x, z^{\prime}(x) \geq$ $0 \Leftrightarrow 1-4 x^{2} \geq 0 \Rightarrow x \leq 1 / 2$. Therefore, $z(x)$ has a maximum point at $x=1 / 2$, so it is not pseudoconvex.

For a real-valued differentiable function, which is said to be a pseudolinear function [21-23], if it is both pseudoconvex and pseudoconcave, similar to the case of real-valued functions, we can also define the following interval-valued pseudolinear functions.

Definition 7. A differentiable interval-valued function $\mathbf{F}_{\mathbf{C}_{v}^{k}}$ : $X \rightarrow \mathscr{I}$, on the convex set $X \subseteq R^{n}$ for given $\mathbf{C}_{v}^{k} \in$ $\mathscr{I}^{k}$, is pseudolinear with respect to $\unlhd_{w}$ on $X$ if it is both pseudoconvex and pseudoconcave on $X$.

This class of pseudolinear interval-valued functions includes many useful functions. For example, the following function is a pseudolinear interval-valued function.

Example 8. The interval-valued function $\mathbf{F}_{\mathbf{C}_{v}^{2}}=\left(1+2 t_{1}\right) x+$ $\left(1+t_{2}\right) \operatorname{In} x: X \rightarrow \mathscr{I}$, where $t=\left(t_{1}, t_{2}\right)^{T}, t_{i} \in[0,1], i=1,2$, and $X=\{x \in R \mid x>0\}$. It can be shown that this intervalvalued function is a pseudolinear interval-valued function.

\section{Characterizations of Pseudolinear Interval-Valued Functions}

In this section, we provide some characterizations of the pseudolinear interval-valued function.

Theorem 9. Let a differentiable interval-valued function $\mathbf{F}_{\mathbf{C}_{v}^{k}}$ : $X \rightarrow \mathscr{I}$, on the convex set $X \subseteq R^{n}$ for given $\mathbf{C}_{v}^{k} \in \mathscr{J}^{k}$. Then, the following statements (i)-(iii) are equivalent.

(i) $\mathbf{F}_{\mathbf{C}_{v}^{k}}$ is pseudolinear over $X$.

(ii) For any $\mathbf{x}$ and $\mathbf{y}$ in $X,(\mathbf{y}-\mathbf{x})^{T} \nabla \mathbf{F}_{\mathbf{C}_{\nu}^{k}}(\mathbf{x})={ }_{w} 0$ if and only if $\mathbf{F}_{\mathbf{C}_{v}^{k}}(\mathbf{y})={ }_{w} \mathbf{F}_{\mathbf{C}_{v}^{k}}(\mathbf{x})$.

(iii) There exists an interval-valued function $\mathbf{P}_{\mathbf{C}^{k}}$ defined on $X \times X$ such that $\left.\mathbf{P}_{\mathbf{C}_{v}^{k}}(\mathbf{x}, \mathbf{y})\right\rangle_{w} 0$ for given $\mathbf{C}_{v}^{k} \in \mathscr{G}^{k}$ and

$$
\mathbf{F}_{\mathbf{C}_{v}^{k}}(\mathbf{y})={ }_{w} \mathbf{F}_{\mathbf{C}_{v}^{k}}(\mathbf{x}) \oplus \mathbf{P}_{\mathbf{C}_{v}^{k}}(\mathbf{x}, \mathbf{y})(\mathbf{y}-\mathbf{x})^{T} \nabla \mathbf{F}_{\mathbf{C}_{v}^{k}}(\mathbf{x})
$$

for any $\mathbf{x}$ and $\mathbf{y}$ in $X$.

Proof. (i) $\Rightarrow$ (ii) Suppose that $\mathbf{F}_{\mathbf{C}_{v}^{k}}$ is pseudolinear intervalvalued function for given $\mathbf{C}_{v}^{k} \in \mathscr{J}^{k}$ on $X$. It can be shown that $\mathbf{F}_{\mathbf{C}_{v}^{k}}(\mathbf{y})={ }_{w} \mathbf{F}_{\mathbf{C}_{v}^{k}}(\mathbf{x})$ implies $(\mathbf{y}-\mathbf{x})^{T} \nabla \mathbf{F}_{\mathbf{C}_{v}^{k}}(\mathbf{x})={ }_{w} 0$. 
Let $\mathbf{x}$ and $\mathbf{y}$ be two points of $X$ such that $\mathbf{F}_{\mathbf{C}_{v}^{k}}(\mathbf{y})={ }_{w} \mathbf{F}_{\mathbf{C}_{v}^{k}}(\mathbf{x})$. For any $\lambda \in(0,1)$, we denote the point $\lambda \mathbf{y}+(1-\lambda) \mathbf{x}$ by $\mathbf{x} \lambda \mathbf{y}$, and it can be shown that $\mathbf{F}_{\mathbf{C}_{v}^{k}}(\mathbf{x} \lambda \mathbf{y})={ }_{w} \mathbf{F}_{\mathbf{C}_{v}^{k}}(\mathbf{x})$.

If $\mathbf{F}_{\mathbf{C}_{v}^{k}}(\mathbf{x} \lambda \mathbf{y}) \succ_{w} \mathbf{F}_{\mathbf{C}_{v}^{k}}(\mathbf{x})$, then $(\mathbf{x}-\mathbf{x} \lambda \mathbf{y})^{T} \nabla \mathbf{F}_{\mathbf{C}_{v}^{k}}(\mathbf{x} \lambda \mathbf{y}) \prec_{w} 0$ since $\mathbf{F}_{\mathbf{C}_{v}^{k}}$ is pseudoconvex for given $\mathbf{C}_{v}^{k} \in \mathscr{I}^{k}$. But $\mathbf{y}-\mathbf{x} \lambda \mathbf{y}=-(1-$ $\lambda)(\mathbf{x}-\mathbf{x} \lambda \mathbf{y}) / \lambda$, and then $(\mathbf{y}-\mathbf{x} \lambda \mathbf{y})^{T} \nabla \mathbf{F}_{\mathbf{C}_{v}^{k}}(\mathbf{x} \lambda \mathbf{y}) \succ_{w} 0$. According to the pseudoconvexity of $\mathbf{F}_{\mathbf{C}_{v}^{k}}$, then $\mathbf{F}_{\mathbf{C}_{v}^{k}}(\mathbf{y}) \geq_{w} \mathbf{F}_{\mathbf{C}_{v}^{k}}(\mathbf{x} \lambda \mathbf{y})$, which contradicts the assumption of $\mathbf{F}_{\mathbf{C}_{v}^{k}}(\mathbf{x} \lambda \mathbf{y})>_{w} \mathbf{F}_{\mathbf{C}_{v}^{k}}(\mathbf{x})={ }_{w} \mathbf{F}_{\mathbf{C}_{v}^{k}}(\mathbf{y})$. So the assumption of $\mathbf{F}_{\mathbf{C}_{\nu}^{k}}(\mathbf{x} \lambda \mathbf{y})>_{w} \mathbf{F}_{\mathbf{C}_{\nu}^{k}}(\mathbf{x})$ is false. Similarly, it can be shown that $\mathbf{F}_{\mathbf{C}_{v}^{k}}(\mathbf{x} \lambda \mathbf{y}) \prec_{w} \mathbf{F}_{\mathbf{C}_{v}^{k}}(\mathbf{x})$ is false by the pseudoconcavity of the interval-valued function $\mathbf{F}_{\mathbf{C}_{v}^{k}}$. Then, we can show that $\mathbf{F}_{\mathbf{C}_{v}^{k}}(\mathbf{x} \lambda \mathbf{y})={ }_{w} \mathbf{F}_{\mathbf{C}_{v}^{k}}(\mathbf{x})$ for given $\mathbf{C}_{v}^{k} \in \mathscr{I}^{k}$ on $X$ and all $\lambda$ in $(0,1)$. So

$$
\lim _{\lambda \rightarrow 0^{+}} \frac{\mathbf{F}_{\mathbf{C}_{v}^{k}}(\mathbf{x} \lambda \mathbf{y}) \ominus \mathbf{F}_{\mathbf{C}_{v}^{k}}(\mathbf{x})}{\lambda}={ }_{w} \nabla \mathbf{F}_{\mathbf{C}_{v}^{k}}(\mathbf{x})^{T}(\mathbf{y}-\mathbf{x})={ }_{w} 0 .
$$

(ii) $\Rightarrow$ (iii) Let the interval-valued function $\mathbf{P}_{\mathbf{C}_{v}^{k}}(\mathbf{x}, \mathbf{y}): X \times$ $Y \rightarrow \mathscr{I}$ defined on $X \times X$, if $\nabla \mathbf{F}_{\mathbf{C}_{v}^{k}}(\mathbf{x})^{T}(\mathbf{y}-\mathbf{x})={ }_{w} 0$ for $\mathbf{x}, \mathbf{y}$ in $X$ and given $\mathbf{C}_{v}^{k} \in \mathscr{J}^{k}$ on $X$, and we define $\mathbf{P}_{\mathbf{C}_{v}^{k}}(\mathbf{x}, \mathbf{y})={ }_{w} 1$ for given $\mathbf{C}_{v}^{k} \in \mathscr{I}^{k}$ on $X$; if $\nabla \mathbf{F}_{\mathbf{C}_{v}^{k}}(\mathbf{x})^{T}(\mathbf{y}-\mathbf{x}) \neq_{w} 0$ for $\mathbf{x}, \mathbf{y}$ in $X$ and given $\mathbf{C}_{v}^{k} \in \mathscr{I}^{k}$ on $X$, we define

$$
\begin{aligned}
\mathbf{P}_{\mathbf{C}_{v}^{k}} & (\mathbf{x}, \mathbf{y}) \\
= & { }_{w} \frac{\mathbf{F}_{\mathbf{C}_{v}^{k}}(\mathbf{y}) \ominus \mathbf{F}_{\mathbf{C}_{v}^{k}}(\mathbf{x})}{\nabla \mathbf{F}_{\mathbf{C}_{v}^{k}}(\mathbf{x})^{T}(\mathbf{y}-\mathbf{x})} \\
= & \left\{\frac{f_{c(t)}(\mathbf{y})-f_{c(t)}(\mathbf{x})}{\nabla f_{c(t)}(\mathbf{x})^{T}(\mathbf{y}-\mathbf{x})} \mid t \in[0,1]^{k}, c(t) \in \mathbf{C}_{v}^{k}\right\} .
\end{aligned}
$$

We can show that $\mathbf{P}_{\mathbf{C}_{v}^{k}}(\mathbf{x}, \mathbf{y}) \succ_{w} 0$ for given $\mathbf{C}_{v}^{k} \in \mathscr{J}^{k}$.

Suppose that $\mathbf{F}_{\mathbf{C}_{v}^{k}}(\mathbf{y})>_{w} \mathbf{F}_{\mathbf{C}_{v}^{k}}(\mathbf{x})$ for given $\mathbf{C}_{v}^{k} \in \mathscr{I}^{k}$. For some $\lambda \in(0,1)$, if $\mathbf{F}_{\mathbf{C}_{v}^{k}}(\mathbf{x} \lambda \mathbf{y}) \preceq_{w} \mathbf{F}_{\mathbf{C}_{v}^{k}}(\mathbf{x})$, using the continuity of $\mathbf{F}_{\mathbf{C}_{v}^{k}}$, there exists $r \in(0,1)$ such that $\lambda \leq r$ and $\mathbf{F}_{\mathbf{C}_{v}^{k}}(\mathbf{x} r \mathbf{y})={ }_{w} \mathbf{F}_{\mathbf{C}_{v}^{k}}(\mathbf{x})$. By (ii),

$$
\lim _{\lambda \rightarrow 0^{+}} \frac{\mathbf{F}_{\mathbf{C}_{v}^{k}}(\mathbf{x} r \mathbf{y}) \ominus \mathbf{F}_{\mathbf{C}_{v}^{k}}(\mathbf{x})}{r}={ }_{w} \nabla \mathbf{F}_{\mathbf{C}_{v}^{k}}(\mathbf{x})^{T}(\mathbf{y}-\mathbf{x})={ }_{w} 0,
$$

which contradicts the assumption of $\nabla \mathbf{F}_{\mathbf{C}_{v}^{k}}(\mathbf{x})^{T}(\mathbf{y}-\mathbf{x}) \neq_{w} 0$ for $\mathbf{x}, \mathbf{y}$ in $X$ and given $\mathbf{C}_{v}^{k} \in \mathscr{J}^{k}$ on $X$. So $\mathbf{F}_{\mathbf{C}_{v}^{k}}(\mathbf{x} \lambda \mathbf{y}) \succ_{w} \mathbf{F}_{\mathbf{C}_{v}^{k}}(\mathbf{x})$ for given $\mathbf{C}_{v}^{k} \in \mathscr{I}^{k}$ and all $\lambda \in(0,1)$; we also have

$$
\lim _{\lambda \rightarrow 0^{+}} \frac{\mathbf{F}_{\mathbf{C}_{v}^{k}}(\mathbf{x} \lambda \mathbf{y}) \ominus \mathbf{F}_{\mathbf{C}_{v}^{k}}(\mathbf{x})}{\lambda}={ }_{w} \nabla \mathbf{F}_{\mathbf{C}_{v}^{k}}(\mathbf{x})^{T}(\mathbf{y}-\mathbf{x}) \succeq_{w} 0,
$$

and since $\nabla \mathbf{F}_{\mathbf{C}_{v}^{k}}(\mathbf{x})^{T}(\mathbf{y}-\mathbf{x}) \neq_{w} 0$ for $\mathbf{x}, \mathbf{y}$ in $X$ and given $\mathbf{C}_{v}^{k} \in \mathscr{J}^{k}$ on $X, \nabla \mathbf{F}_{\mathbf{C}_{v}^{k}}(\mathbf{x})^{T}(\mathbf{y}-\mathbf{x}) \succ_{w} 0$ holds. It can be shown that

$$
\mathbf{P}_{\mathbf{C}_{v}^{k}}(\mathbf{x}, \mathbf{y})={ }_{w} \frac{\mathbf{F}_{\mathbf{C}_{v}^{k}}(\mathbf{y}) \ominus \mathbf{F}_{\mathbf{C}_{v}^{k}}(\mathbf{x})}{\nabla \mathbf{F}_{\mathbf{C}_{v}^{k}}(\mathbf{x})^{T}(\mathbf{y}-\mathbf{x})} \succ_{w} 0
$$

for given $\mathbf{C}_{v}^{k} \in \mathscr{I}^{k}$ on $X$.
Similarly, we can show that $\mathbf{P}_{\mathbf{C}_{v}^{k}}(\mathbf{x}, \mathbf{y}) \succ_{w} 0$ if $\mathbf{F}_{\mathbf{C}_{v}^{k}}(\mathbf{y}) \prec_{w}$ $\mathbf{F}_{\mathbf{C}_{v}^{k}}(\mathbf{x})$ for given $\mathbf{C}_{v}^{k} \in \mathscr{I}^{k}$.

(iii) $\Rightarrow$ (i) It is obvious that

$$
\mathbf{F}_{\mathbf{C}_{v}^{k}}(\mathbf{y})={ }_{w} \mathbf{F}_{\mathbf{C}_{v}^{k}}(\mathbf{x}) \oplus \mathbf{P}_{\mathbf{C}_{v}^{k}}(\mathbf{x}, \mathbf{y})(\mathbf{y}-\mathbf{x})^{T} \nabla \mathbf{F}_{\mathbf{C}_{v}^{k}}(\mathbf{x})
$$

for any $\mathbf{x}$ and $\mathbf{y}$ in $X$, which implies $(\mathbf{y}-\mathbf{x})^{T} \nabla \mathbf{F}_{\mathbf{C}_{v}^{k}}(\mathbf{x})={ }_{w} 0$ if and only if $\mathbf{F}_{\mathbf{C}_{v}^{k}}(\mathbf{y})={ }_{w} \mathbf{F}_{\mathbf{C}_{v}^{k}}(\mathbf{x})$.

Theorem 10. Let $\mathbf{F}_{\mathbf{C}_{v}^{k}}: X \rightarrow \mathscr{I}$ be an interval-valued function defined on the convex set $X \subseteq R^{n}$ for given $\mathbf{C}_{v}^{k} \in \mathscr{I}^{k}$, which is once continuously differentiable on $X$. Then $\mathbf{F}_{\mathbf{C}_{v}^{k}}$ is pseudolinear on $X$ if and only if $(\mathbf{y}-\mathbf{x})^{T} \nabla \mathbf{F}_{\mathbf{C}_{v}^{k}}(\mathbf{x})={ }_{w} 0$ implies $\mathbf{F}_{\mathbf{C}_{v}^{k}}(\mathbf{x})={ }_{w} \mathbf{F}_{\mathbf{C}_{v}^{k}}(\mathbf{x} \lambda \mathbf{y})$ for any $\mathbf{x}$ and $\mathbf{y}$ in $X$ and any $\lambda \in[0,1]$.

Proof. Suppose that $\mathbf{F}_{\mathbf{C}_{v}^{k}}$ is pseudolinear on $X$. Let $\mathbf{x}$ and $\mathbf{y}$ in $X$ be such that $(\mathbf{y}-\mathbf{x})^{T} \nabla \mathbf{F}_{\mathbf{C}_{v}^{k}}(\mathbf{x})={ }_{w} 0$ for given $\mathbf{C}_{v}^{k} \in \mathscr{I}^{k}$. Then, for any $\lambda \in[0,1]$,

$$
(\mathbf{x} \lambda \mathbf{y}-\mathbf{x})^{T} \nabla \mathbf{F}_{\mathbf{C}_{v}^{k}}(\mathbf{x})={ }_{w} \lambda(\mathbf{y}-\mathbf{x})^{T} \nabla \mathbf{F}_{\mathbf{C}_{v}^{k}}(\mathbf{x})={ }_{w} 0 .
$$

By (ii) of Theorem $9, \mathbf{F}_{\mathbf{C}_{v}^{k}}(\mathbf{x})={ }_{w} \mathbf{F}_{\mathbf{C}_{v}^{k}}(\mathbf{x} \lambda \mathbf{y})$ for any $\mathbf{x}$ and $\mathbf{y}$ in $X$ and any $\lambda \in[0,1]$.

Conversely, suppose that $(\mathbf{y}-\mathbf{x})^{T} \nabla \mathbf{F}_{\mathbf{C}_{v}^{k}}(\mathbf{x})={ }_{w} 0$ implies $\mathbf{F}_{\mathbf{C}_{v}^{k}}(\mathbf{x})={ }_{w} \mathbf{F}_{\mathbf{C}_{v}^{k}}(\mathbf{x} \lambda \mathbf{y})$ for any $\mathbf{x}$ and $\mathbf{y}$ in $X$ and any $\lambda \in[0,1]$. If $\mathbf{F}_{\mathbf{C}_{v}^{k}}$ is not pseudolinear on $X$, then there exist $\mathbf{x}$ and $\mathbf{y}$ in $X$ such that $\mathbf{F}_{\mathbf{C}_{v}^{k}}(\mathbf{x})={ }_{w} \mathbf{F}_{\mathbf{C}_{v}^{k}}(\mathbf{y})$, but $(\mathbf{y}-\mathbf{x})^{T} \nabla \mathbf{F}_{\mathbf{C}_{v}^{k}}(\mathbf{x}) \neq_{w} 0$, which means that $(\mathbf{y}-\mathbf{x})^{T} \nabla \mathbf{F}_{\mathbf{C}_{v}^{k}}(\mathbf{x})={ }_{w} 0$ and $\mathbf{F}_{\mathbf{C}_{v}^{k}}(\mathbf{x})={ }_{w} \mathbf{F}_{\mathbf{C}_{v}^{k}}(\mathbf{y})$ cannot occur. Suppose that $(\mathbf{y}-\mathbf{x})^{T} \nabla \mathbf{F}_{\mathbf{C}_{v}^{k}}(\mathbf{x}) \succ_{w} 0$, which means $(\mathbf{y}-\mathbf{x})^{T} \nabla f_{c(t)}(\mathbf{x})>0$ for $c(t) \in \mathbf{C}_{v}^{k}$. We define $f(\lambda)=$ $\mathbf{F}_{\mathbf{C}_{v}^{k}}(\mathbf{x} \lambda \mathbf{y})$, which is once continuously differentiable function. According to the assumption, $f(0)=f(1)$ and $f^{\prime}(0)=$ $\left\{(\mathbf{y}-\mathbf{x})^{T} \nabla f_{c(t)}(\mathbf{x})>0 \mid \forall c(t) \in \mathbf{C}_{v}^{k}\right\}$. So $f(\lambda)$ assumes a local maximum at some point $\lambda_{0} \in(0,1)$. Then

$$
f^{\prime}\left(\lambda_{0}\right)=(\mathbf{y}-\mathbf{x})^{T} \nabla \mathbf{F}_{\mathbf{C}_{v}^{k}}\left(\mathbf{x} \lambda_{0} \mathbf{y}\right)=0 .
$$

So $\left(\mathbf{x} \lambda_{0} \mathbf{y}-\mathbf{x}\right)^{T} \nabla \mathbf{F}_{\mathbf{C}_{v}^{k}}\left(\mathbf{x} \lambda_{0} \mathbf{y}\right)=\lambda_{0}(\mathbf{y}-\mathbf{x})^{T} \nabla \mathbf{F}_{\mathbf{C}_{v}^{k}}\left(\mathbf{x} \lambda_{0} \mathbf{y}\right)=0$. By the assumption, $\mathbf{F}_{\mathbf{C}_{v}^{k}}(\mathbf{x})={ }_{w} \mathbf{F}_{\mathbf{C}_{v}^{k}}(\mathbf{x} \lambda \mathbf{y})$ for all $0 \leq \lambda \leq \lambda_{0}$, and then $(\mathbf{y}-\mathbf{x})^{T} \nabla \mathbf{F}_{\mathbf{C}_{v}^{k}}(\mathbf{x})=0$, which is a contradiction.

How to check for interval pseudolinearity can be shown by the following example.

Example 11. Consider the interval-valued function on $X=$ $\{\pi / 2>x>0\}:$

$$
\begin{aligned}
& \mathbf{F}_{\mathbf{C}_{v}^{2}}(\mathbf{x})=\left\{f_{c(t)}(x)=\left(2+t_{1}\right) x\right. \\
& \left.\quad+\left(1+t_{2}\right) \sin x \mid \forall t_{1}, t_{2} \in[0,1]\right\} .
\end{aligned}
$$

From (ii) of Theorem 9, for any $x$ and $y$ in $X,(\mathbf{y}-$ $\mathbf{x})^{T} \nabla \mathbf{F}_{\mathbf{C}_{v}^{2}}(\mathbf{x})={ }_{w} 0$ means $(y-x)^{T} \nabla f_{c(t)}(x)=(y-x)\left[\left(2+t_{1}\right)+\right.$ $\left.\left(1+t_{2}\right) \cos x\right]=0$ for all $t_{1}, t_{2} \in[0,1]$. Since $\nabla f_{c(t)}(x)=$ $\left(2+t_{1}\right)+\left(1+t_{2}\right) \cos x \neq 0$, if and only if $x=y$, so, for $f_{c(t)}(x)=$ 
$\left(2+t_{1}\right) x+\left(1+t_{2}\right) \sin x$ and $f_{c(t)}(y)=\left(2+t_{1}\right) y+\left(1+t_{2}\right) \sin y$, we have $f_{c(t)}(x)=f_{c(t)}(y)$ for all $t_{1}, t_{2} \in[0,1]$, which implies $\mathbf{F}_{\mathbf{C}_{v}^{2}}(\mathbf{y})={ }_{w} \mathbf{F}_{\mathbf{C}_{v}^{2}}(\mathbf{x})$.

Therefore, the interval-valued function is a pseudolinear interval-valued function.

The following interval-valued linear fractional function is a class of interval-valued pseudolinear functions:

$$
\mathbf{F}_{\mathbf{C}_{v}^{k}}(\mathbf{x})=\frac{\mathbf{A}^{T} \mathbf{x}+C}{\mathbf{B}^{T} \mathbf{x}+D}
$$

where $\mathbf{x} \in R^{n}$, $\mathbf{A}$ and $\mathbf{B}$ are all in $\mathscr{I}^{n}$, and $C$ and $D$ are interval number. We can show that $\mathbf{F}_{\mathbf{C}_{v}^{k}}(\mathbf{x})$ is pseudolinear on $S_{F}=$ $\left\{\mathbf{x} \in R^{n} \mid \mathbf{B}^{T} \mathbf{x}+D \succ_{w} 0\right\}$,

$$
\mathbf{F}_{\mathbf{C}_{v}^{k}}(\mathbf{y}) \ominus \mathbf{F}_{\mathbf{C}_{v}^{k}}(\mathbf{x})={ }_{w} \frac{\mathbf{B}^{T} \mathbf{x}+D}{\mathbf{B}^{T} \mathbf{y}+D}(\mathbf{y}-\mathbf{x})^{T} \nabla \mathbf{F}_{\mathbf{C}_{v}^{k}}(\mathbf{x}),
$$

where $\mathbf{P}_{\mathbf{C}_{v}^{k}}(\mathbf{x}, \mathbf{y})=\left(\mathbf{B}^{T} \mathbf{x}+D\right) /\left(\mathbf{B}^{T} \mathbf{y}+D\right) \succ_{w} 0$ if $\mathbf{x}$ and $\mathbf{y}$ are in $S_{F}$.

For interval-valued linear fractional function, we have

$$
\mathbf{P}_{\mathbf{C}_{v}^{k}}(\mathbf{x}, \mathbf{y}) \mathbf{P}_{\mathbf{C}_{v}^{k}}(\mathbf{y}, \mathbf{z})=\mathbf{P}_{\mathbf{C}_{v}^{k}}(\mathbf{x}, \mathbf{z})
$$

for any $\mathbf{x}, \mathbf{y}$, and $\mathbf{z}$. It can be shown that (29) implies $\mathbf{P}_{\mathbf{C}_{v}^{k}}(\mathbf{x}, \mathbf{x})=1$ and $\mathbf{P}_{\mathbf{C}_{v}^{k}}(\mathbf{x}, \mathbf{y})=1 / \mathbf{P}_{\mathbf{C}_{v}^{k}}(\mathbf{y}, \mathbf{x})$ for any $\mathbf{x}$ and $\mathbf{y}$ in $S_{F}$. We can show the following conclusion.

Theorem 12. Let a pseudolinear interval-valued function $\mathbf{F}_{\mathrm{C}_{v}^{k}}$ : $X \rightarrow \mathscr{I}$, on the convex set $X \subseteq R^{n}$ with proportional functional $\mathbf{P}_{\mathbf{C}_{v}^{k}}(\mathbf{x}, \mathbf{y})$ for given $\mathbf{C}_{v}^{k} \in \mathcal{I}^{k}$. If $\mathbf{P}_{\mathbf{C}_{v}^{k}}(\mathbf{x}, \mathbf{y})$ satisfies condition (29) and $\mathbf{P}_{\mathbf{C}_{v}^{k}}(\mathbf{x}, \cdot)$ is differentiable for each $\mathbf{x}$ in $X$, then there exists an interval-valued linear fractional function $\mathbf{G}_{\mathbf{C}_{v}^{k}}$ such that $X \subset S_{G}$ and $\mathbf{G}_{\mathbf{C}_{v}^{k}}(\mathbf{x})={ }_{w} \mathbf{F}_{\mathbf{C}_{v}^{k}}(\mathbf{x})$ for all $\mathbf{x}$ in $X$.

Proof. Suppose that $\mathbf{F}_{\mathrm{C}^{k}}$ is not constant on $X$ and $X$ contains more than one point. Take $\mathbf{y}$ and $\mathbf{z}$ in $X$ such that $\mathbf{F}_{\mathrm{C}_{v}^{k}}(\mathbf{z}) \neq$ $\mathbf{F}_{\mathbf{C}_{v}^{k}}(\mathbf{y})$. Then

$$
\mathbf{F}_{\mathbf{C}_{v}^{k}}(\mathbf{x})={ }_{w} \mathbf{F}_{\mathbf{C}_{v}^{k}}(\mathbf{y}) \oplus \mathbf{P}_{\mathbf{C}_{v}^{k}}(\mathbf{y}, \mathbf{x})(\mathbf{x}-\mathbf{y})^{T} \nabla \mathbf{F}_{\mathbf{C}_{v}^{k}}(\mathbf{y})
$$

for any $\mathbf{x}$ and $\mathbf{y}$ in $X$.

Since $\mathbf{P}_{\mathbf{C}_{v}^{k}}(\mathbf{x}, \cdot)$ is differentiable for each $\mathbf{x}$ in $X$, then

$$
\begin{aligned}
\nabla \mathbf{F}_{\mathbf{C}_{v}^{k}}(\mathbf{x})={ }_{w} \mathbf{P}_{\mathbf{C}_{v}^{k}}(\mathbf{y}, \mathbf{x}) \nabla \mathbf{F}_{\mathbf{C}_{v}^{k}}(\mathbf{y}) \\
\\
\quad \oplus(\mathbf{x}-\mathbf{y})^{T} \nabla \mathbf{F}_{\mathbf{C}_{v}^{k}}(\mathbf{y}) \nabla_{2} \mathbf{P}_{\mathbf{C}_{v}^{k}}(\mathbf{y}, \mathbf{x})
\end{aligned}
$$

for any $\mathbf{x}$ and $\mathbf{y}$ in $X$, where $\nabla_{2} \mathbf{P}$ denotes the gradient of $\mathbf{P}$ with respect to the second variable.
From (30), we have

$$
\begin{aligned}
& \mathbf{F}_{\mathbf{C}_{v}^{k}}(\mathbf{x}) \ominus \mathbf{F}_{\mathbf{C}_{v}^{k}}(\mathbf{z})={ }_{w} \mathbf{P}_{\mathbf{C}_{v}^{k}}(\mathbf{z}, \mathbf{x}) \nabla \mathbf{F}_{\mathbf{C}_{v}^{k}}(\mathbf{z})^{T}(\mathbf{x}-\mathbf{z}), \\
& \mathbf{F}_{\mathbf{C}_{v}^{k}}(\mathbf{x}) \ominus \mathbf{F}_{\mathbf{C}_{v}^{k}}(\mathbf{z}) \\
& { }_{{ }_{w}} \mathbf{F}_{\mathbf{C}_{v}^{k}}(\mathbf{y}) \oplus \mathbf{P}_{\mathbf{C}_{v}^{k}}(\mathbf{y}, \mathbf{x}) \nabla \mathbf{F}_{\mathbf{C}_{v}^{k}}(\mathbf{y})^{T}(\mathbf{x}-\mathbf{y}) \\
& \quad \ominus\left[\mathbf{F}_{\mathbf{C}_{v}^{k}}(\mathbf{y}) \oplus \mathbf{P}_{\mathbf{C}_{v}^{k}}(\mathbf{y}, \mathbf{z}) \nabla \mathbf{F}_{\mathbf{C}_{v}^{k}}(\mathbf{y})^{T}(\mathbf{z}-\mathbf{y})\right] \\
& ={ }_{w} \mathbf{P}_{\mathbf{C}_{v}^{k}}(\mathbf{y}, \mathbf{x}) \nabla \mathbf{F}_{\mathbf{C}_{v}^{k}}(\mathbf{y})^{T}(\mathbf{x}-\mathbf{y}) \\
& \quad \ominus \mathbf{P}_{\mathbf{C}_{v}^{k}}(\mathbf{y}, \mathbf{z}) \nabla \mathbf{F}_{\mathbf{C}_{v}^{k}}(\mathbf{y})^{T}(\mathbf{z}-\mathbf{y}) .
\end{aligned}
$$

By (31), we have

$$
\begin{aligned}
\mathbf{P}_{\mathbf{C}_{v}^{k}}(\mathbf{z}, \mathbf{x}) \nabla \mathbf{F}_{\mathbf{C}_{v}^{k}}(\mathbf{z})^{T}(\mathbf{x}-\mathbf{z})={ }_{w} \mathbf{P}_{\mathbf{C}_{v}^{k}}(\mathbf{z}, \mathbf{x}) \\
\cdot\left[\mathbf{P}_{\mathbf{C}_{v}^{k}}(\mathbf{y}, \mathbf{z}) \nabla \mathbf{F}_{\mathbf{C}_{v}^{k}}(\mathbf{y})\right. \\
\left.\oplus(\mathbf{z}-\mathbf{y})^{T} \nabla \mathbf{F}_{\mathbf{C}_{v}^{k}}(\mathbf{y}) \nabla_{2} \mathbf{P}_{\mathbf{C}_{v}^{k}}(\mathbf{y}, \mathbf{z})\right]^{T}(\mathbf{x}-\mathbf{z}),
\end{aligned}
$$

By (32)-(33), we have

$$
\begin{aligned}
& {\left[\mathbf{P}_{\mathbf{C}_{v}^{k}}(\mathbf{y}, \mathbf{x}) \ominus \mathbf{P}_{\mathbf{C}_{v}^{k}}(\mathbf{y}, \mathbf{z})\right] \nabla \mathbf{F}_{\mathbf{C}_{v}^{k}}(\mathbf{y})^{T}(\mathbf{z}-\mathbf{y})} \\
& \quad{ }_{w} \mathbf{P}_{\mathbf{C}_{v}^{k}}(\mathbf{y}, \mathbf{x}) \nabla \mathbf{F}_{\mathbf{C}_{v}^{k}}(\mathbf{y})^{T}[(\mathbf{x}-\mathbf{y})-(\mathbf{x}-\mathbf{z})] \\
& \quad \ominus \mathbf{P}_{\mathbf{C}_{v}^{k}}(\mathbf{y}, \mathbf{z}) \nabla \mathbf{F}_{\mathbf{C}_{v}^{k}}(\mathbf{y})^{T}(\mathbf{z}-\mathbf{y}) \\
& ={ }_{w}\left[\mathbf{P}_{\mathbf{C}_{v}^{k}}(\mathbf{z}, \mathbf{x}) \nabla \mathbf{F}_{\mathbf{C}_{v}^{k}}(\mathbf{y})^{T}(\mathbf{z}-\mathbf{y}) \nabla_{2} \mathbf{P}_{\mathbf{C}_{v}^{k}}(\mathbf{y}, \mathbf{z})\right]^{T} \\
& \cdot(\mathbf{x}-\mathbf{z}) .
\end{aligned}
$$

Since $\mathbf{F}_{\mathbf{C}_{v}^{k}}(\mathbf{z}) \neq \mathbf{F}_{\mathbf{C}_{v}^{k}}(\mathbf{y})$, we have $\nabla \mathbf{F}_{\mathbf{C}_{v}^{k}}(\mathbf{y})^{T}(\mathbf{z}-\mathbf{y}) \neq 0$. From (34), we have

$$
\begin{aligned}
\mathbf{P}_{\mathbf{C}_{v}^{k}} & (\mathbf{y}, \mathbf{x}) \ominus \mathbf{P}_{\mathbf{C}_{v}^{k}}(\mathbf{y}, \mathbf{z}) \\
\quad={ }_{w} & {\left[\mathbf{P}_{\mathbf{C}_{v}^{k}}(\mathbf{z}, \mathbf{x}) \nabla_{2} \mathbf{P}_{\mathbf{C}_{v}^{k}}(\mathbf{y}, \mathbf{z})\right]^{T}(\mathbf{x}-\mathbf{z}) . }
\end{aligned}
$$

Multiplying (35) by $\mathbf{P}_{\mathrm{C}_{v}^{k}}(\mathbf{x}, \mathbf{y})$, we obtain

$$
1 \ominus \mathbf{P}_{\mathbf{C}_{v}^{k}}(\mathbf{x}, \mathbf{z})={ }_{w}\left[\mathbf{P}_{\mathbf{C}_{v}^{k}}(\mathbf{z}, \mathbf{y}) \nabla_{2} \mathbf{P}_{\mathbf{C}_{v}^{k}}(\mathbf{y}, \mathbf{z})\right]^{T}(\mathbf{x}-\mathbf{z}) .
$$

So

$$
\begin{aligned}
\mathbf{P}_{\mathbf{C}_{v}^{k}}(\mathbf{z}, \mathbf{x}) & ={ }_{w} \frac{1}{\mathbf{P}_{\mathbf{C}_{v}^{k}}(\mathbf{x}, \mathbf{z})} \\
& ={ }_{w}\left[1 \ominus \mathbf{P}_{\mathbf{C}_{v}^{k}}(\mathbf{z}, \mathbf{y}) \nabla_{2} \mathbf{P}_{\mathbf{C}_{v}^{k}}(\mathbf{y}, \mathbf{z})^{T}(\mathbf{x}-\mathbf{z})\right]^{-1} .
\end{aligned}
$$

Substituting (37) into (30) and replacing y by $\mathbf{z}$, it can be shown that

$$
\begin{aligned}
& \mathbf{F}_{\mathbf{C}_{v}^{k}}(\mathbf{x})={ }_{w} \mathbf{F}_{\mathbf{C}_{v}^{k}}(\mathbf{z}) \oplus(\mathbf{x}-\mathbf{z})^{T} \nabla \mathbf{F}_{\mathbf{C}_{v}^{k}}(\mathbf{z}) \\
& \cdot\left[1 \ominus \mathbf{P}_{\mathbf{C}_{v}^{k}}(\mathbf{z}, \mathbf{y}) \nabla_{2} \mathbf{P}_{\mathbf{C}_{v}^{k}}(\mathbf{y}, \mathbf{z})^{T}(\mathbf{x}-\mathbf{z})\right]^{-1}
\end{aligned}
$$

is an interval-valued linear fractional function $\mathbf{G}_{\mathbf{C}_{v}^{k}}$. 
The result of Theorem 12 and (iii) of Theorem 10 can be shown by the following example.

Example 13. Consider the interval-valued linear fractional function

$$
\mathbf{F}_{\mathbf{C}_{v}^{1}}(\mathbf{x})=\frac{\mathbf{A}^{T} \mathbf{x}+C}{\mathbf{B}^{T} \mathbf{x}+D},
$$

where $\mathbf{x} \in R_{+}, \mathbf{A}^{T}=[2,3]=2+t_{1}$, and $\mathbf{B}^{T}=[3,4]=3+t_{2}$ are all in $\mathscr{I}^{2}$ and $C=[0,1]=t_{3}$ and $D=[1,2]=1+t_{4}$ are interval number, where $t_{i} \in[0,1], i=1,2,3,4$.

We denote that

$$
\begin{aligned}
\mathbf{P}_{\mathbf{C}_{v}^{1}} & (\mathbf{x}, \mathbf{y}) \\
& =\frac{\mathbf{B}^{T} \mathbf{x}+D}{\mathbf{B}^{T} \mathbf{y}+D} \\
& =\left\{\frac{\left(3+t_{2}\right) x+\left(1+t_{4}\right)}{\left(3+t_{2}\right) y+\left(1+t_{4}\right)}>0 \mid \forall t_{i} \in[0,1]\right\} .
\end{aligned}
$$

It can be shown that $\mathbf{P}_{\mathbf{C}_{v}^{1}}(\mathbf{x}, \mathbf{y})$ satisfies (29), and the following is satisfied:

$$
\mathbf{F}_{\mathbf{C}_{v}^{1}}(\mathbf{y})={ }_{w} \mathbf{F}_{\mathbf{C}_{v}^{1}}(\mathbf{x}) \oplus \mathbf{P}_{\mathbf{C}_{v}^{1}}(\mathbf{x}, \mathbf{y})(\mathbf{y}-\mathbf{x})^{T} \nabla \mathbf{F}_{\mathbf{C}_{v}^{1}}(\mathbf{x})
$$

for any $\mathbf{x}$ and $\mathbf{y}$ in $R_{+}$. From (iii) of Theorem 9, the intervalvalued function $\mathbf{F}_{\mathbf{C}_{v}^{1}}(\mathbf{x})$ is a pseudolinear interval-valued function.

According to Theorem 12, there exists an intervalvalued linear fractional function $\mathbf{G}_{\mathbf{C}_{v}^{1}}(\mathbf{x})={ }_{w} \mathbf{F}_{\mathbf{C}_{v}^{1}}(\mathbf{z}) \oplus(\mathbf{x}-$ $\mathbf{z})^{T} \nabla \mathbf{F}_{\mathbf{C}_{v}^{1}}(\mathbf{z})\left[1 \quad \ominus \mathbf{P}_{\mathbf{C}_{v}^{1}}(\mathbf{z}, \mathbf{y}) \nabla_{2} \mathbf{P}_{\mathbf{C}_{v}^{1}}(\mathbf{y}, \mathbf{z})^{T}(\mathbf{x}-\mathbf{z})\right]^{-1}$, such that $\mathbf{G}_{\mathbf{C}_{v}^{1}}(\mathbf{x})={ }_{w} \mathbf{F}_{\mathbf{C}_{v}^{1}}(\mathbf{x})$.

For all $t_{i} \in[0,1], i=1,2,3,4$, we have

$$
\begin{aligned}
& {\left[1 \quad \ominus \mathbf{P}_{\mathbf{C}_{v}^{1}}(\mathbf{z}, \mathbf{y}) \nabla_{2} \mathbf{P}_{\mathbf{C}_{v}^{1}}(\mathbf{y}, \mathbf{z})^{T}(\mathbf{x}-\mathbf{z})\right]^{-1}=\{1} \\
& -\frac{\left(3+t_{2}\right) z+\left(1+t_{4}\right)}{\left(3+t_{2}\right) y+\left(1+t_{4}\right)} \\
& \quad \cdot\left\{-\frac{\left(3+t_{2}\right)\left[\left(3+t_{2}\right) y+\left(1+t_{4}\right)\right]}{\left[\left(3+t_{2}\right) z+\left(1+t_{4}\right)\right]^{2}}\right\}(x-z) \mid \forall t_{i} \\
& \quad \in[0,1]\}=\left\{\frac{\left(3+t_{2}\right) x+\left(1+t_{4}\right)}{\left(3+t_{2}\right) z+\left(1+t_{4}\right)} \mid \forall t_{i} \in[0,1]\right\} \\
& ={ }_{w} \mathbf{P}_{\mathbf{C}_{v}^{1}}(\mathbf{x}, \mathbf{z}) .
\end{aligned}
$$

Then, we get

$$
\mathbf{G}_{\mathbf{C}_{v}^{1}}(\mathbf{x})={ }_{w} \mathbf{F}_{\mathbf{C}_{v}^{1}}(\mathbf{z}) \oplus(\mathbf{x}-\mathbf{z})^{T} \nabla \mathbf{F}_{\mathbf{C}_{v}^{1}}(\mathbf{z}) \mathbf{P}_{\mathbf{C}_{v}^{1}}(\mathbf{x}, \mathbf{z}) .
$$

By (iii) of Theorem 9, we have $\mathbf{G}_{\mathbf{C}_{v}^{1}}(\mathbf{x})={ }_{w} \mathbf{F}_{\mathbf{C}_{v}^{1}}(\mathbf{x})$.

\section{The Solution Sets of Interval-Valued Pseudolinear Programs}

In this section, we consider the following interval-valued programming:

$$
\begin{array}{ll}
(\mathrm{IVOP}) \min & \mathbf{F}_{\mathrm{C}_{v}^{k}}(\mathbf{x}) \\
\text { subject to } & \mathbf{x}=\left(x_{1}, \ldots, x_{n}\right) \in X \subseteq R^{n},
\end{array}
$$

where $\mathbf{F}_{\mathrm{C}_{v}^{k}}(\mathbf{x}): X \rightarrow \mathscr{I}$ is an interval-valued function for given $\mathbf{C}_{v}^{k} \in \mathscr{I}^{k}$ and the feasible set $X$ is a convex subset of $R^{n}$. We denote the solution set of (IVOP) as

$$
\bar{S}=\underset{\mathbf{x} \in X}{\arg \min } \mathbf{F}_{\mathbf{C}_{v}^{k}}(\mathbf{x}),
$$

which is nonempty. If $\mathbf{F}_{\mathbf{C}_{v}^{k}}(\mathbf{x})$ is pseudolinear, then the solution set $\bar{S}$ of (IVOP) is a convex set.

The following characterization of the solution set of the problem of (IVOP) can be obtained.

Theorem 14. Let $\mathbf{F}_{\mathrm{C}_{v}^{k}}(\mathbf{x}): X \rightarrow \mathscr{I}$ be a pseudolinear intervalvalued function for given $\mathbf{C}_{v}^{k} \in \mathscr{I}^{k}$ and $\overline{\mathbf{x}} \in \bar{S}$. Then $\bar{S}=\widetilde{S}=$ $\widehat{S}=\breve{S}$, where

$$
\begin{aligned}
& \widetilde{S}=\left\{\mathbf{x} \in X \mid(\overline{\mathbf{x}}-\mathbf{x})^{T} \nabla \mathbf{F}_{\mathrm{C}_{v}^{k}}(\mathbf{x})={ }_{w} 0\right\}, \\
& \widehat{S}=\left\{\mathbf{x} \in X \mid(\overline{\mathbf{x}}-\mathbf{x})^{T} \nabla \mathbf{F}_{\mathrm{C}_{v}^{k}}(\overline{\mathbf{x}})={ }_{w} 0\right\}, \\
& \breve{S}=\left\{\mathbf{x} \in X \mid(\overline{\mathbf{x}}-\mathbf{x})^{T} \nabla \mathbf{F}_{\mathrm{C}_{v}^{k}}(\mathbf{x} \lambda \overline{\mathbf{x}})={ }_{w} 0, \quad \forall \lambda \in[0,1]\right\} .
\end{aligned}
$$

Proof. It is obvious that $\mathbf{x} \in \bar{S}$ if and only if $\mathbf{F}_{\mathbf{C}_{v}^{k}}(\mathbf{x})={ }_{w} \mathbf{F}_{\mathbf{C}_{v}^{k}}(\overline{\mathbf{x}})$, which implies $f_{c(t)}(\mathbf{x})=f_{c(t)}(\overline{\mathbf{x}})$ for $t \in[0,1]^{k}$ and $c(t) \in \mathbf{C}_{v}^{k}$. From (ii) of Theorem $9, \mathbf{F}_{\mathbf{C}_{v}^{k}}(\mathbf{x})={ }_{w} \mathbf{F}_{\mathbf{C}_{v}^{k}}(\overline{\mathbf{x}})$ if and only if

$$
(\overline{\mathbf{x}}-\mathbf{x})^{T} \nabla \mathbf{F}_{\mathbf{C}_{v}^{k}}(\mathbf{x})={ }_{w} 0={ }_{w}(\overline{\mathbf{x}}-\mathbf{x})^{T} \nabla \mathbf{F}_{\mathbf{C}_{v}^{k}}(\overline{\mathbf{x}}) .
$$

Therefore, $\bar{S}=\widetilde{S}=\widehat{S}$.

It can be seen that $\breve{S} \subset \widetilde{S}=\bar{S}$. Let $\mathbf{x} \in \bar{S}=\widetilde{S}$, and then

$$
(\overline{\mathbf{x}}-\mathbf{x})^{T} \nabla \mathbf{F}_{\mathbf{C}_{v}^{k}}(\mathbf{x})={ }_{w} 0 .
$$

For each $\lambda \in(0,1)$, we get

$$
(\overline{\mathbf{x}}-\mathbf{x} \lambda \overline{\mathbf{x}})^{T} \nabla \mathbf{F}_{\mathbf{C}_{v}^{k}}(\mathbf{x})={ }_{w}(1-\lambda)(\overline{\mathbf{x}}-\mathbf{x})^{T} \nabla \mathbf{F}_{\mathbf{C}_{v}^{k}}(\mathbf{x})={ }_{w} 0 .
$$

Therefore, from (ii) of Theorem 9, we have

$$
\mathbf{F}_{\mathbf{C}_{v}^{k}}(\mathbf{x})={ }_{w} \mathbf{F}_{\mathbf{C}_{v}^{k}}(\mathbf{x} \lambda \overline{\mathbf{x}})
$$

By the pseudolinearity of $\mathbf{F}_{\mathbf{C}_{v}^{k}}(\mathbf{x})$ and (52), we obtain

$$
(\overline{\mathbf{x}}-\mathbf{x})^{T} \nabla \mathbf{F}_{\mathbf{C}_{v}^{k}}(\mathbf{x} \lambda \overline{\mathbf{x}})={ }_{w} 0, \quad \forall \lambda \in(0,1) .
$$

So $\mathbf{x} \in \breve{S}$ and $\bar{S} \subset \breve{S}$. Thus, $\bar{S}=\breve{S}$. 
Corollary 15. Let $\mathbf{F}_{\mathbf{C}_{v}^{k}}(\mathbf{x}): X \rightarrow \mathscr{I}$ be a pseudolinear interval-valued function for given $\mathbf{C}_{v}^{k} \in \mathscr{I}^{k}$ and $\overline{\mathbf{x}} \in \bar{S}$. Then $\bar{S}=\widetilde{S}^{*}=\widehat{S}^{*}=\breve{S}^{*}$, where

$$
\begin{aligned}
\widetilde{S}^{*} & =\left\{\mathbf{x} \in X \mid(\overline{\mathbf{x}}-\mathbf{x})^{T} \nabla \mathbf{F}_{\mathbf{C}_{v}^{k}}(\mathbf{x}) \succeq_{w} 0\right\}, \\
\widehat{S}^{*} & =\left\{\mathbf{x} \in X \mid(\overline{\mathbf{x}}-\mathbf{x})^{T} \nabla \mathbf{F}_{\mathbf{C}_{v}^{k}}(\overline{\mathbf{x}}) \succeq_{w} 0\right\}, \\
\breve{S}^{*} & =\left\{\mathbf{x} \in X \mid(\overline{\mathbf{x}}-\mathbf{x})^{T} \nabla \mathbf{F}_{\mathbf{C}_{v}^{k}}(\mathbf{x} \lambda \overline{\mathbf{x}}) \succeq_{w} 0, \quad \forall \lambda \in[0,1]\right\} .
\end{aligned}
$$

Proof. From (46), it is clear that $\bar{S} \subset \widetilde{S}^{*}$. Let $\mathbf{x} \in \widetilde{S}^{*}$; that is, for $\mathbf{x} \in X$,

$$
(\overline{\mathbf{x}}-\mathbf{x})^{T} \nabla \mathbf{F}_{\mathbf{C}_{v}^{k}}(\mathbf{x}) \succeq_{w} 0 .
$$

Then, from (iii) of Theorem 9 and the pseudolinearity of $\mathbf{F}_{\mathbf{C}_{v}^{k}}(\mathbf{x})$, we have

$$
\begin{aligned}
\mathbf{F}_{\mathbf{C}_{v}^{k}}(\overline{\mathbf{x}})={ }_{w} \mathbf{F}_{\mathbf{C}_{v}^{k}}(\mathbf{x}) & \\
& \oplus \mathbf{P}_{\mathbf{C}_{v}^{k}}(\mathbf{x}, \overline{\mathbf{x}})(\overline{\mathbf{x}}-\mathbf{x})^{T} \nabla \mathbf{F}_{\mathbf{C}_{v}^{k}}(\mathbf{x}) \succeq_{w} \mathbf{F}_{\mathbf{C}_{v}^{k}}(\mathbf{x}) .
\end{aligned}
$$

Then $\mathbf{x} \in \bar{S}$, which implies $\bar{S}=\widetilde{S}^{*}$. It can be also similarly proved that $\bar{S}=\widehat{S}^{*}$. that

We can also prove that $\bar{S}=\breve{S}^{*}$ as follows. In fact, it is clear

$$
\bar{S}=\breve{S} \subset \breve{S}^{*} \subset \widetilde{S}^{*}=\bar{S},
$$

where $\breve{S}$ is defined by (48). Thus, the conclusion follows.

Example 16. Consider the following pseudolinear intervalvalued program:

$$
\begin{aligned}
\min & \mathbf{F}_{\mathbf{C}_{v}^{2}}(\mathbf{x}) \\
& =\left\{\left(2+t_{1}\right) x+\left(1+t_{2}\right) \sin x \mid \forall t_{i} \in[0,1], i=1,2\right\}
\end{aligned}
$$

subject to $x \in X \subseteq R$,

where $\mathbf{F}_{\mathrm{C}^{2}}(\mathbf{x}): X \rightarrow \mathscr{I}$ is an interval-valued function, $t=\left(t_{1}, t_{2}\right)^{T}, t_{i} \in[0,1], i=1,2$, and $X=\{x \in R \mid \pi / 2>x>$ $0\}$. By Example 11, $\mathbf{F}_{\mathrm{C}_{v}^{2}}(\mathbf{x})$ is a pseudolinear interval-valued function and $\bar{S}=\{0\}$.

According to Theorem 14, we get $\bar{S}=\widetilde{S}=\widehat{S}=\breve{S}$.

\section{Conclusions}

In this paper, we introduced the concept of the pseudolinear interval-valued functions. We have obtained some basic characterizations of an interval-valued pseudolinear function. By means of the properties of interval-valued pseudolinearity, a class of interval-valued pseudolinear programs was considered, and the solution set of the interval-valued pseudolinear optimization problem was characterized.

\section{Conflict of Interests}

The authors declare that there is no conflict of interests regarding the publication of this paper.

\section{Acknowledgments}

This work was partially supported by Natural Science Basic Research Plan in Shaanxi Province of China (Program nos. 2013JQ1020, 2013KJXX-29, and 2014 JM8307), National Natural Science Foundation of China (Program nos. 11301415, 61100166, 61303092, 11401469, 11426176, and 11401357), special funds for the construction of key disciplines funded projects in Shaanxi Province, project funded by China Postdoctoral Science Foundation (no. 2014M552453), the National Key Technologies R\&D Program of China under Grant no. 2012BAH16F02. Hanzhong Administration of Science and Technology under Grant no. 2013hzzx-39, and the Science Plan Foundations of the Education Bureau of Shaanxi Province (nos. 11JK1051, 2013JK1098, 2013JK1130, 2013JK1182, and 14JK1661).

\section{References}

[1] C. Jiang, X. Han, G. R. Liu, and G. P. Liu, "A nonlinear interval number programming method for uncertain optimization problems," European Journal of Operational Research, vol. 188, no. 1, pp. 1-13, 2008.

[2] S. Chanas and D. Kuchta, "Multiobjective programming in optimization of interval objective functions-a generalized approach," European Journal of Operational Research, vol. 94, no. 3, pp. 594-598, 1996.

[3] S.-T. Liu, "Posynomial geometric programming with interval exponents and coefficients," European Journal of Operational Research, vol. 186, no. 1, pp. 17-27, 2008.

[4] H.-C. Wu, "The Karush-KUHn-Tucker optimality conditions in an optimization problem with interval-valued objective function," European Journal of Operational Research, vol. 176, no. 1, pp. 46-59, 2007.

[5] H. C. Wu, "Duality theory for optimization problems with interval-valued objective functions," Journal of Optimization Theory and Applications, vol. 144, no. 3, pp. 615-628, 2010.

[6] Y. H. Sun and L. S. Wang, "Optimality conditions and duality in nondifferentiable interval-valued programming," Journal of Industrial and Management Optimization, vol. 9, no. 1, pp. 131142,2013

[7] A. Jayswal, I. Stancu-Minasian, and I. Ahmad, "On sufficiency and duality for a class of interval-valued programming problems," Applied Mathematics and Computation, vol. 218, no. 8, pp. 4119-4127, 2011.

[8] Y. Chalco-Cano, W. A. Lodwick, and A. Rufian-Lizana, "Optimality conditions of type KKT for optimization problem with interval-valued objective function via generalized derivative," Fuzzy Optimization and Decision Making, vol. 3, no. 12, pp. 305322, 2013

[9] A. K. Bhurjee and G. Panda, "Efficient solution of interval optimization problem," Mathematical Methods of Operations Research, vol. 76, no. 3, pp. 273-288, 2012.

[10] A. K. Bhurjee and G. Panda, "Multi-objective interval fractional programming problems : an approach for obtaining efficient solutions," OPSEARCH, vol. 52, no. 1, pp. 156-167, 2015.

[11] M. Jana and G. Panda, "Solution of nonlinear interval vector optimization problem," Operational Research, vol. 14, no. 1, pp. 71-85, 2014.

[12] J. K. Zhang, S. Y. Liu, L. F. Li, and Q. X. Feng, "The KKT optimality conditions in a class of generalized convex optimization 
problems with an interval-valued objective function," Optimization Letters, vol. 8, no. 2, pp. 607-631, 2014.

[13] M. Fiedler, J. Nedoma, J. Ramík, J. Rohn, and K. Zimmermann, Linear Optimization Problems with Inexact Data, Springer, New York, NY, USA, 2006.

[14] M. Hladík, "Optimal value bounds in nonlinear programming with interval data," TOP, vol. 19, no. 1, pp. 93-106, 2011.

[15] M. Hladík, "How to determine basis stability in interval linear programming," Optimization Letters, vol. 8, no. 1, pp. 375-389, 2014.

[16] M. Hladík, "On approximation of the best case optimal value in interval linear programming," Optimization Letters, vol. 8, no. 7, pp. 1985-1997, 2014.

[17] W. Li, X. Liu, and H. Li, "Generalized solutions to interval linear programmes and related necessary and sufficient optimality conditions," Optimization Methods and Software, 2014.

[18] W. Li, J. Luo, Q. Wang, and Y. Li, "Checking weak optimality of the solution to linear programming with interval right-hand side," Optimization Letters, vol. 8, no. 4, pp. 1287-1299, 2014.

[19] J. Luo, W. Li, and Q. Wang, "Checking strong optimality of interval linear programming with inequality constraints and nonnegative constraints," Journal of Computational and Applied Mathematics, vol. 260, pp. 180-190, 2014.

[20] O. L. Mangasarian, "Pseudo-convex functions," SIAM Journal on Control and Optimization, vol. 3, pp. 281-290, 1965.

[21] K. L. Chew and E. V. Choo, "Pseudolinearity and efficiency," Mathematical Programming, vol. 28, no. 2, pp. 226-239, 1984.

[22] V. Jeyakumar and X. Q. Yang, "On characterizing the solution sets of pseudolinear programs," Journal of Optimization Theory and Applications, vol. 87, no. 3, pp. 747-755, 1995.

[23] S. Komlósi, "First and second order characterizations of pseudolinear functions," European Journal of Operational Research, vol. 67, no. 2, pp. 278-286, 1993. 


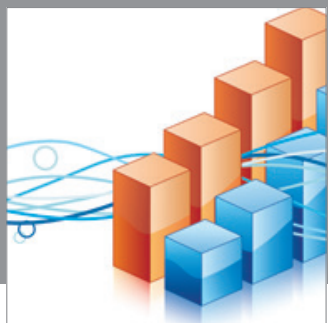

Advances in

Operations Research

mansans

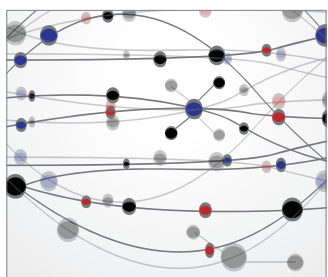

The Scientific World Journal
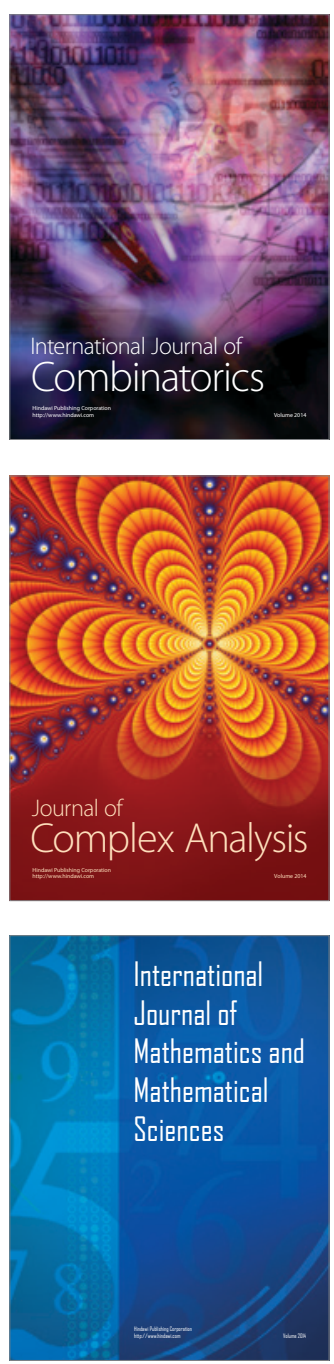
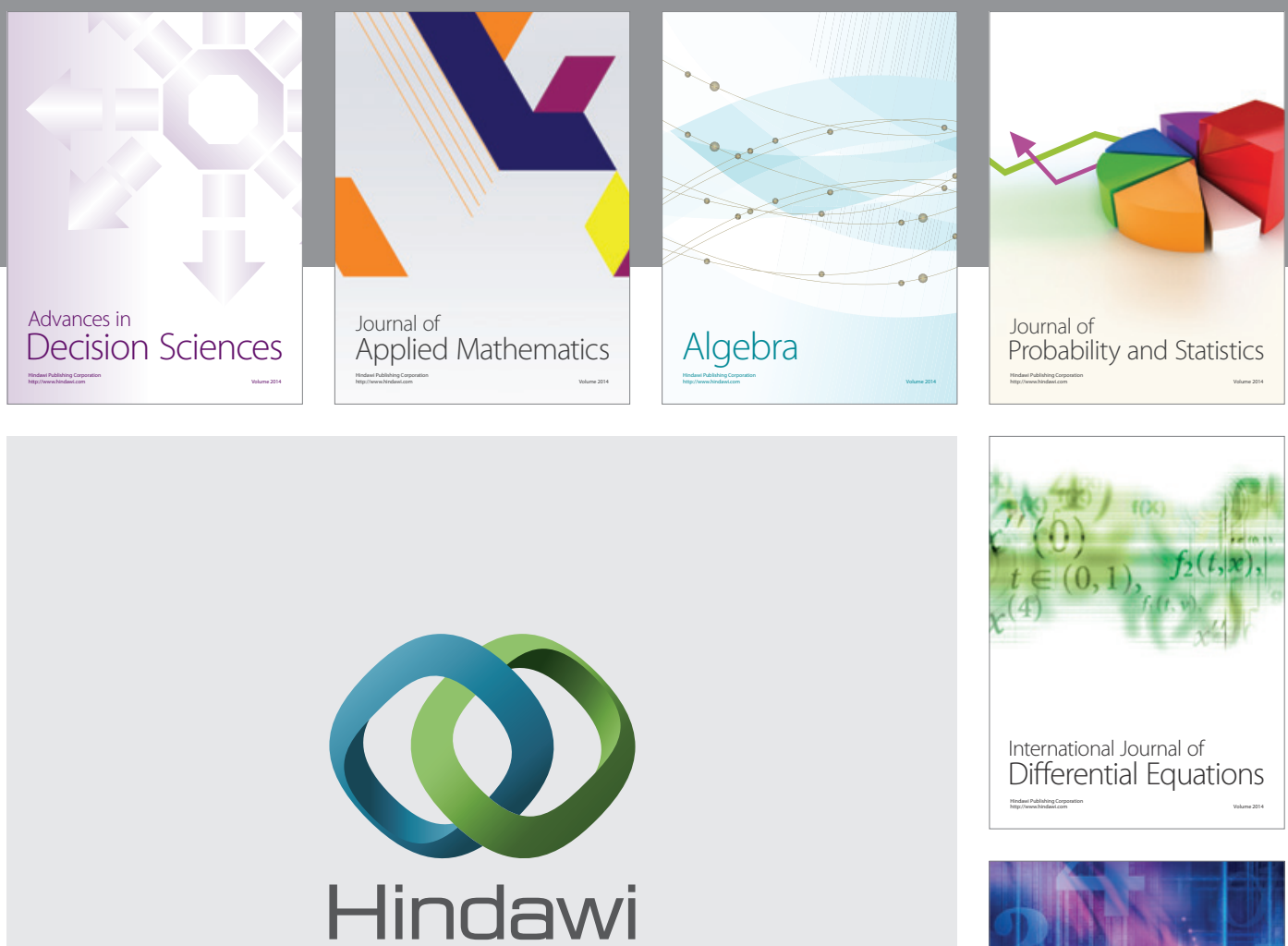

Submit your manuscripts at http://www.hindawi.com
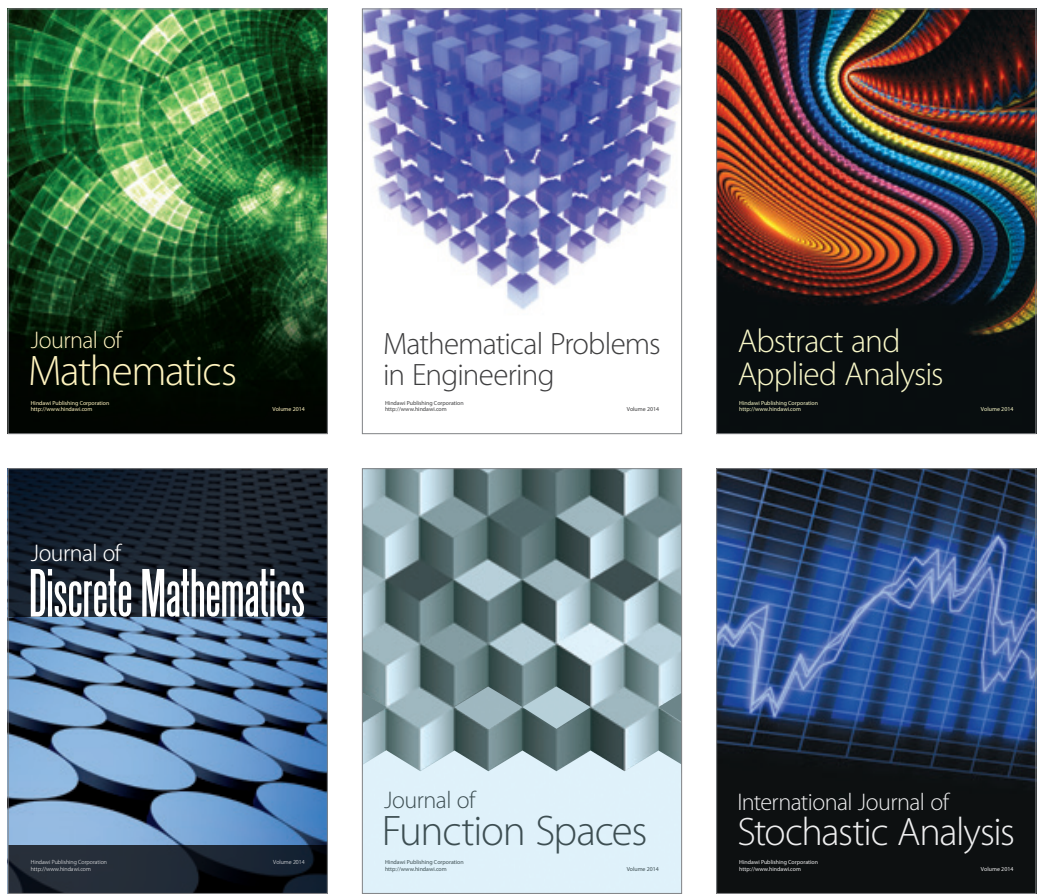

Journal of

Function Spaces

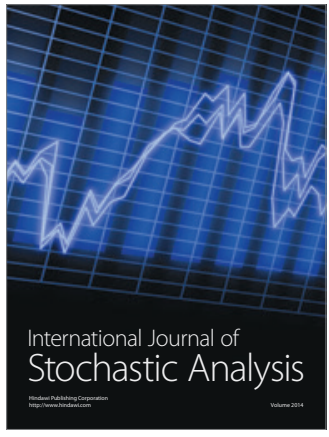

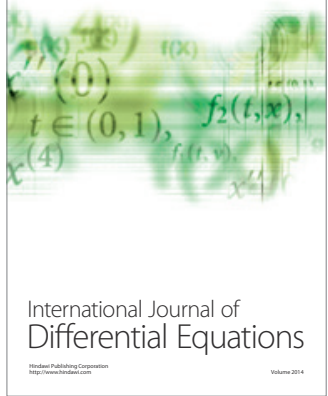
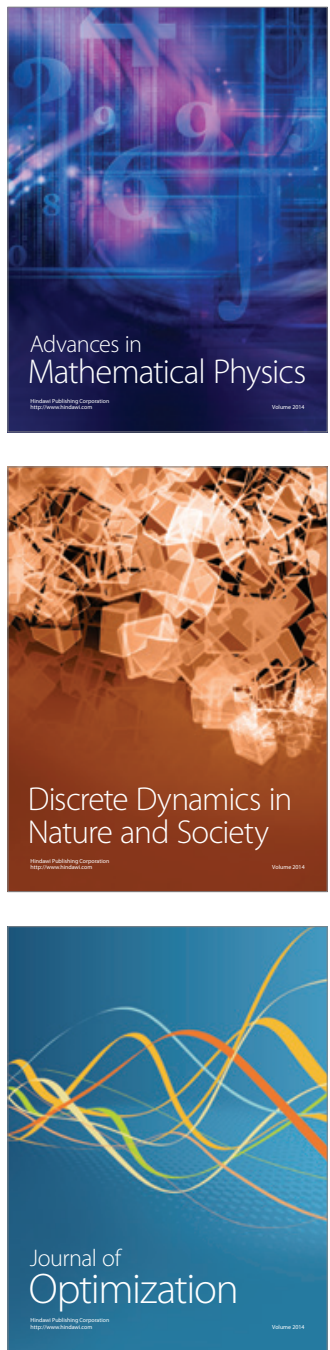